\title{
Observing Radio Pulsars in the Galactic Centre with the Square Kilometre Array
}

\author{
R. P. Eatough ${ }^{* 1,2}$, T. J. W. Lazio ${ }^{3,4}$, J. Casanellas ${ }^{5}$, S. Chatterjee ${ }^{6}$, J. M. Cordes $^{6}$,

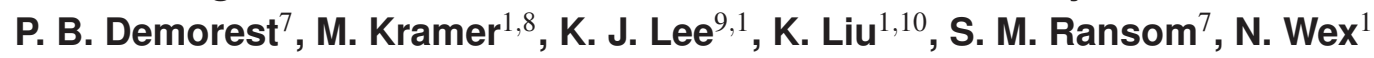 \\ ${ }^{1}$ Max-Planck-Institut für Radioastronomie, Bonn, 53121, Germany; ${ }^{2}$ E-mail: reatough@mpif $\mathrm{r}$-bonn.mpg. de; \\ ${ }^{3}$ Jet Propulsion Laboratory, California Institute of Technology, M/S 138-308, 4800 Oak Grove Dr, Pasadena, CA 91109, \\ USA; ${ }^{4}$ E-mail: joseph. lazio@ jpl . nasa.gov; ${ }^{5}$ Max Planck Institute for Gravitational Physics (Albert Einstein \\ Institute), Science Park Potsdam-Golm, Am Mühlenberg 1, D-14476, Germany; ${ }^{6}$ Department of Astronomy and Space \\ Sciences, Cornell University, Ithaca, NY 14853, USA, ${ }^{7}$ National Radio Astronomy Observatory, 520 Edgemont Road, \\ Charlottesville, Virginia 22903-2475, USA; ${ }^{8}$ Jodrell Bank Centre for Astrophysics, Alan Turing Building, University of \\ Manchester, Manchester, M13 9PL, UK; ${ }^{9}$ Kavli institute for Astronomy and Astrophysics, Peking University, Beijing \\ 100871, P. R. China,; ${ }^{10}$ Station de radioastronomie de Nançay, Observatoire de Paris, CNRS/INSU, Université \\ d'Orléans, F-18330 Nançay, France.
}

The discovery and timing of radio pulsars within the Galactic centre is a fundamental aspect of the SKA Science Case, responding to the topic of "Strong Field Tests of Gravity with Pulsars and Black Holes" (Kramer et al. 2004; Cordes et al. 2004). Pulsars have in many ways proven to be excellent tools for testing the General theory of Relativity and alternative gravity theories (see Wex (2014) for a recent review). Timing a pulsar in orbit around a companion, provides a unique way of probing the relativistic dynamics and spacetime of such a system. The strictest tests of gravity, in strong field conditions, are expected to come from a pulsar orbiting a black hole. In this sense, a pulsar in a close orbit $\left(P_{\text {orb }}<1 \mathrm{yr}\right)$ around our nearest supermassive black hole candidate, Sagittarius $A^{*}$ - at a distance of $\simeq 8.3 \mathrm{kpc}$ in the Galactic centre (Gillessen et al. 2009a) - would be the ideal tool. Given the size of the orbit and the relativistic effects associated with it, even a slowly spinning pulsar would allow the black hole spacetime to be explored in great detail (Liu et al. 2012). For example, measurement of the frame dragging caused by the rotation of the supermassive black hole, would allow a test of the "cosmic censorship conjecture." The "no-hair theorem" can be tested by measuring the quadrupole moment of the black hole. These are two of the prime examples for the fundamental studies of gravity one could do with a pulsar around Sagittarius A*. As will be shown here, SKA1-MID and ultimately the SKA will provide the opportunity to begin to find and time the pulsars in this extreme environment.

Advancing Astrophysics with the Square Kilometre Array

June 8-13, 2014

Giardini Naxos, Italy

\footnotetext{
* Speaker.
} 


\section{Background}

A combination of radio and infrared (IR) astrometric observations has established the presence of a dark mass in the Galactic centre (GC), whose properties are most consistent with being a supermassive black hole (BH) (Eckart \& Genzel 1996; Ghez et al. 1998; Reid \& Brunthaler 2004; Ghez et al. 2008; Gillessen et al. 2009a,b). Known by its radio nomenclature of Sagittarius A* (Sgr $A^{*}$ ), current estimates are that the mass of this BH is $4.30 \pm 0.20_{\text {(stat) }} \pm 0.30_{\text {(sys) }} \times 10^{6} \mathrm{M}_{\odot}$ (Gillessen et al. 2009a). Monitoring the orbits of the closest stars S0-2 (Schödel et al. 2002) and S0-102 (Meyer et al. 2012) could provide constraints on the extent to which their orbits are perturbed which in turn would constrain the enclosed mass, which could be stars, dark matter, or both (Sabha et al. 2012; Gillessen et al. 2009b). Current work with the Keck Telescope, the VLT and with the VLT interferometer (VLTI) and proposed work with extremely large telescopes (ELTs; e.g. the European ELT and the GSMT) will lead to IR astrometric precisions of 0.5 mas with single apertures and $10 \mu$ as with the VLTI and the GRAVITY instrument (Weinberg et al. 2005; Eisenhauer et al. 2011). These resolutions allow orbital perturbations of 4.15 and 0.08 au, respectively, to be measured that will arise from General Relativity (GR) and from Newtonian effects caused by other stars and dark matter. It is expected that source confusion in IR surveys with ELTs will limit the sample of stars to those with orbits larger than about 200 to $300 \mathrm{au} ; \sim 100$ stars with semi-major axes $\lesssim 3000$ au for a 30-m ELT (Weinberg et al. 2005).

The discovery and timing of radio pulsars orbiting Sgr A*, and within the central stellar cluster, will provide a powerful means to test GR, as well as probe the dark matter content and magnetoionic medium within the nucleus of our Galaxy. An SKA radio pulsar program can yield much more precise localizations on objects arbitrarily close to the innermost stable circular orbit (ISCO, radius $\gtrsim 0.1 \mathrm{au}$, depending on the spin of the Sgr $\mathrm{A}^{*} \mathrm{BH}$ ). Confusion limits do not apply to the time-varying signals of pulsars. In comparison with the best forecasted precisions of IR astrometry and Doppler measurements, pulsar timing has the potential of increasing the testing and diagnostic precision by at least three orders of magnitude. Pulsars offer this prospect because timing precisions correspond to spatial localizations (from ranging) smaller than a light second ( $0.2 \mu$ as) compared to the localizations expected from the best proposed IR interferometry, of no better than $10 \mu$ as $(0.1 \mathrm{au})$.

\section{The pulsar population in the GC}

Several lines of argument suggest that not only is there a large neutron star (NS) population in the GC but that many NSs should be active radio pulsars. Figure 1 (left panel) shows schematically the contents of the GC as determined both empirically and from theoretical arguments about galaxy formation. Ghez et al. (2008) and Gillessen et al. (2009b) show that the stellar cluster around Sgr $A^{*}$ consists mainly of early-type stars, whose short lifetimes $\left(\sim 10^{7} \mathrm{yr}\right)$ means that either massive star formation in the region is ongoing or that there has been a starburst of order $6 \mathrm{Myr}$ ago, although the presence of young, massive stars in a region where tidal forces should inhibit star formation present a conundrum: "the paradox of youth" - (Ghez et al. 2003). Nonetheless, stars with masses of 10-20 $\mathrm{M}_{\odot}$ are NS progenitors, and the presence of a population of young massive stars near Sgr A* suggests that there must exist a numerous NS population as well. The time scales 
Matter Content of the GC

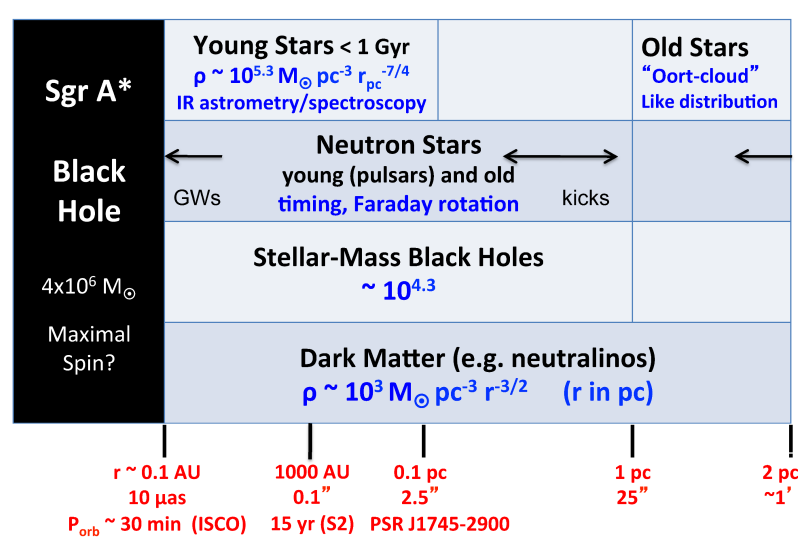

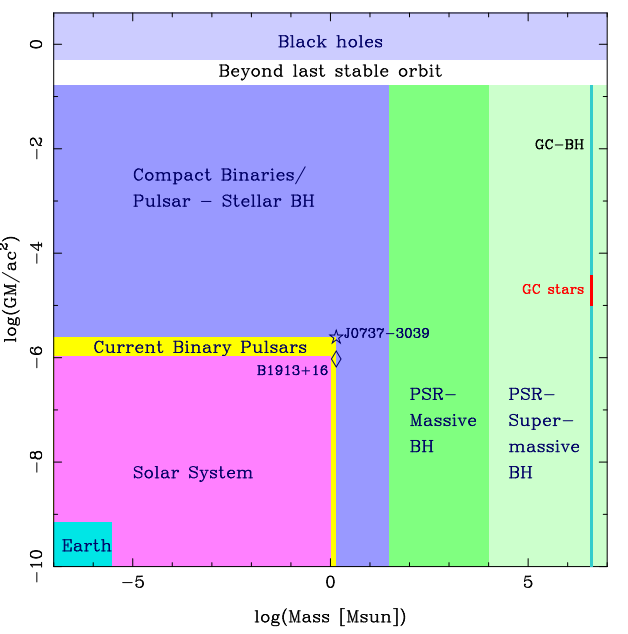

Figure 1: (Left) Schematic view of the matter content of the GC. Young stars (e.g., S0-2) are known to approach as close as $0.1^{\prime \prime}(1000 \mathrm{AU})$ to Sgr A*. The combination of young, hot stars in the GC and mass segregation suggests that there are likely to be compact stellar remnants (NSs and BHs) at least within 1000 au, and potentially much closer. Finally, there could also be dark matter particles. The SKA, operating at frequencies near $10 \mathrm{GHz}$, will search for NS, in the form of active radio pulsars, as close as possible to Sgr A*. (Right) Depth of gravitational potential $\left(G M / a c^{2}\right)$ probed by an orbit of semi-major axis $a$ as a function of the mass of the binary system $M$. The regions probed by solar system tests and known pulsars in binary systems are indicated, with the double neutron star systems PSR B1913+16 and PSR J0737-3039 shown explicitly. The vertical cyan line indicates the mass of Sgr A* (GC-BH). The red line segment shows the region probed by the $\mathrm{S}$ stars in the GC. It is clear that substantially deeper gravitational potentials (larger $G M / a c^{2}$ ) remains to be probed, and would be accessible to radio pulsar observations (Kramer et al. 2004).

also work out for many of these NSs to be active radio pulsars: radio emission of canonical $10^{12} \mathrm{G}$ objects lasts for $\sim 10 \mathrm{Myr}$, the time scale for spindown to lengthen spin periods past the "death line" of a few seconds.

Estimates of the supernova rate within $100 \mathrm{pc}$ of Sgr $\mathrm{A}^{*}$ based on the number of young stars in the region and on the heating of X-ray gas range from $10^{-3} \mathrm{yr}^{-1}$ to $10^{-2} \mathrm{yr}^{-1}$ and are consistent, after accounting for the fraction of supernovae that produce pulsars, with the number of point radio sources in the region that could be pulsars (Lazio \& Cordes 2008). Such agreement is coarse but suggestive that there are of order $10^{3}$ to $10^{4}$ active pulsars in the region, of which about 200 to 2000 (20\%) would be beamed toward the Earth. More recently, and by combining results from the GC radio point source counts described above, the detected GC pulsar population at that time, non-detections in high-frequency pulsar surveys of the inner parsec, radio and gammaray measurements of diffuse emission, infra-red observations of massive stellar GC populations, pulsar wind nebulae candidates, and the supernova rates from X-ray data, Wharton et al. (2012) estimate that $\sim 10^{3}$ active radio pulsars in the inner parsec should be beamed toward Earth.

Pulsars in the GC, like other stellar populations, will orbit the central BH Sgr A* mostly as isolated objects but some will be in binaries with companions of all types: main-sequence and post-main-sequence stars, white dwarfs, NSs, or even BHs (Faucher-Giguère \& Loeb 2011). A population of "recycled" pulsars (i.e., NSs spun up to millisecond periods by accretion) is also expected because the stellar density in the GC is large enough so that tidal capture and exchange 


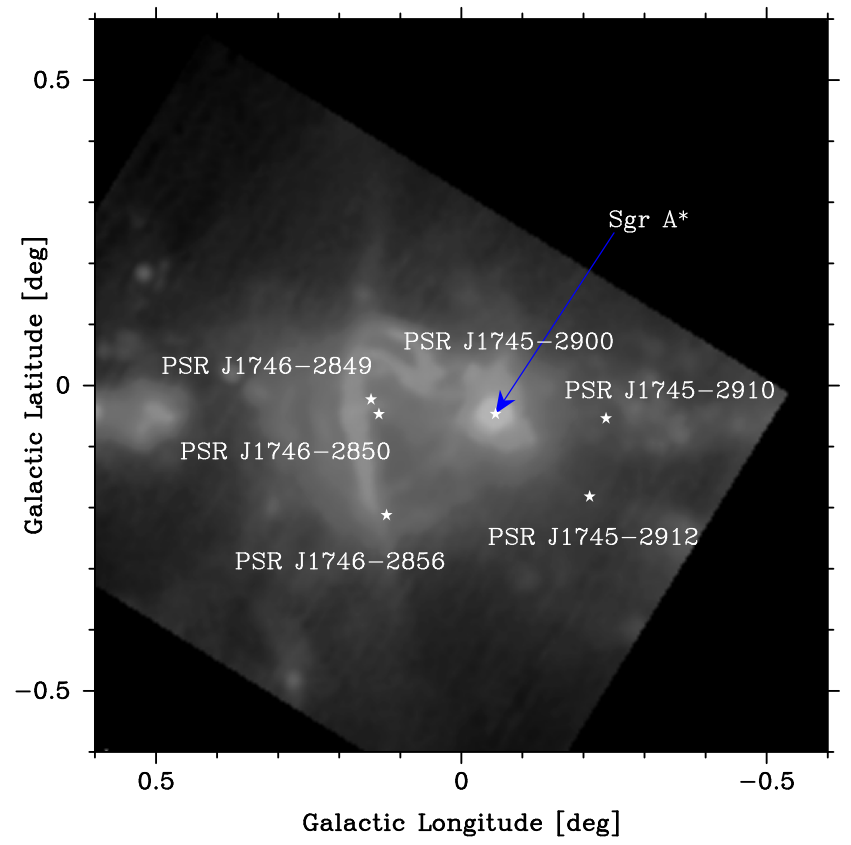

Figure 2: The positions of detected radio pulsars in the central $0.5^{\circ}$ toward the $\mathrm{GC}$ overlaid on a $10.55 \mathrm{GHz}$ continuum map made with the Effelsberg telescope (Seiradakis et al. 1989). Assuming a GC distance of $8.3 \mathrm{kpc}, 0.5^{\circ}$ corresponds to a projected distance of $\sim 70 \mathrm{pc}$. Even PSR J1745-2900, which overlaps the position of Sgr $\mathrm{A}^{*}$ on this scale $\left(\sim 3^{\prime \prime} \equiv 0.1 \mathrm{pc}\right.$ offset $)$, is too distant for gravity tests. Figure courtesy of B. Klein.

reactions should occur as they do in globular clusters. Voss \& Gilfanov (2007) show that tidal capture of NSs by low-mass main-sequence stars is the dominant mechanism for forming binary NS in approximately the central $1^{\prime}$ of M31 and these can produce millisecond pulsars (MSPs) via long-term accretion. Along with similarly captured stellar-mass BHs, accreting objects in the central part of M31 can account for the excess number of point X-ray sources in the inner bulge of M31. The same processes should occur in the GC over a similar-sized region $(0.1-0.2 \mathrm{kpc}$ ) (Muno et al. 2005).

Arguably the strongest evidence for a significant NS population in the central parsec now comes from the recent detection of a radio loud magnetar - PSR J1745-2900, an inherently rare class of pulsar, just $\sim 3^{\prime \prime}(0.1 \mathrm{pc}$ ) from Sgr A* (Kennea et al. 2013; Mori et al. 2013; Eatough et al. 2013a; Shannon \& Johnston 2013). Along with the five pulsars previously discovered in proximity to Sgr A* (at $10^{\prime}-15^{\prime}$ - see Figure 2 (Johnston et al. 2006; Deneva et al. 2009)) these objects cannot be explained as part of a Galactic disk population because the number of foreground pulsars expected in the pulsar surveys to date is much less than one. Consequently, the question of the GC pulsar population has been recently revisited by a number of authors (Chennamangalam \& Lorimer 2014; Zhang et al. 2014; Dexter \& O'Leary 2014). As will be described in Section 3, it is pulsars in compact orbits $\left(P_{\mathrm{orb}}<1 \mathrm{yr}\right.$ - corresponding to a semi-major axis of $\sim 160 \mathrm{au}$ ) around the $\mathrm{BH}$ that will enable the most precise tests of GR. Recent simulations suggest that up $\sim 200$ pulsars could orbit within 4000 au of the $\mathrm{BH}$, the closest of which could have a semi-major axis of $\sim 120$ au (Zhang et al. 2014). It is only through observations with the SKA that the full GC pulsar 
population (whatever its size) will be detected.

Certain dark matter models predict that dark matter particles can efficiently accumulate inside compact objects such as NS, eventually causing them to collapse into a BH. The GC pulsar population, as detected by the SKA, can thus also be used to place stringent constraints on dark matter models, with older pulsars and MSPs being the most constraining (Bramante \& Linden 2014).

\section{Testing gravity with GC pulsars}

With their large moments of inertia and fast spin rates, radio pulsars are excellent clocks. The clocklike property of pulsars orbiting Sgr A* will provide unique probes of the spacetime, plasma and dark matter around the massive central BH and are a powerful probe of GR (Paczynski \& Trimble 1979; Wex et al. 1996; Pfahl \& Loeb 2004) itself. As we show below, GR and other perturbations of GC pulsars are large enough so that even rather "noisy" pulsars, relative to typical precision timing requirements, will do the job.

Pulse times-of-arrival (TOAs) from an orbiting pulsar can be described in terms of the standard Keplerian parameters and in a model-independent parameterized post-Newtonian (PPN) formalism. Any deviations from the PPN values expected from GR would provide strong evidence of a post-GR theory of gravity. The most recent illustration of this method of testing theories of gravity is from the double pulsar PSR J0737-3039A/B, for which the measured PPN parameters indicate agreement with GR at better than the $1 \%$ level (Kramer \& Stairs 2008). However, PSR J0737-3039A/B still represents a relatively weak field case. Consider the depth of the gravitational potential to be $G M / a c^{2}$, for a total system mass $M$ and semi-major axis $a$, with $G$ and $c$ being the usual physical constants. The current population of binary pulsars probes only to $G M / a c^{2} \lesssim 10^{-5.5}$. By contrast, a pulsar with an orbit comparable to the star S0-2 $\left.\approx 15 \mathrm{yr}\right)$ would probe to $G M / a c^{2} \sim 10^{-4.5}$; pulsars with shorter orbital periods could probe to even larger values of $G M / a c^{2}$ (Figure 1). Star-star interactions of course can cause perturbations that make GR tests more difficult.

For a pulsar in a close orbit $\left(P_{\text {orb }}<1 \mathrm{yr}\right)$ around Sgr A* one expects to see a number of relativistic effects, like time dilation, Shapiro delay and relativistic peri-center precession, all of them with a magnitude, that as we mention above, does not even require a particularly high timing precision to measure them with excellent accuracy (Liu et al. 2012). The time dilation, for instance, is expected to be of order 10 to 20 minutes. Consequently, soon after the discovery relativistic effects will be well measured, give the mass of Sgr A* with high precision, and allow for the first gravity tests. After a few orbits, one will be able to separate the Lense-Thirring drag from the orbital motion and measure the spin of the $\mathrm{BH}$ with a fractional precision of $10^{-2}$ to $10^{-3}$ (Liu et al. 2012). A first test is to see if the dimensionless spin-parameter does not exceed one, as expected for a Kerr BH in GR (cosmic censorship conjecture - see Liu et al. (2012)). The quadrupole moment of Sgr A* leads to a characteristic signature in the timing residuals, directly related to the quadrupolar structure of its potential (Figure 3). By this, the effect of the quadrupole can uniquely be identified in the motion of the pulsar, and its magnitude can be measured with good to very good precision, allowing for a test of the no-hair theorem due to the unique relation of mass, spin and quadrupole for a Kerr solution (Wex \& Kopeikin 1999; Liu et al. 2012). Figure 4 illustrates some of the relativistic effects that could be determined from measuring pulse TOAs of 


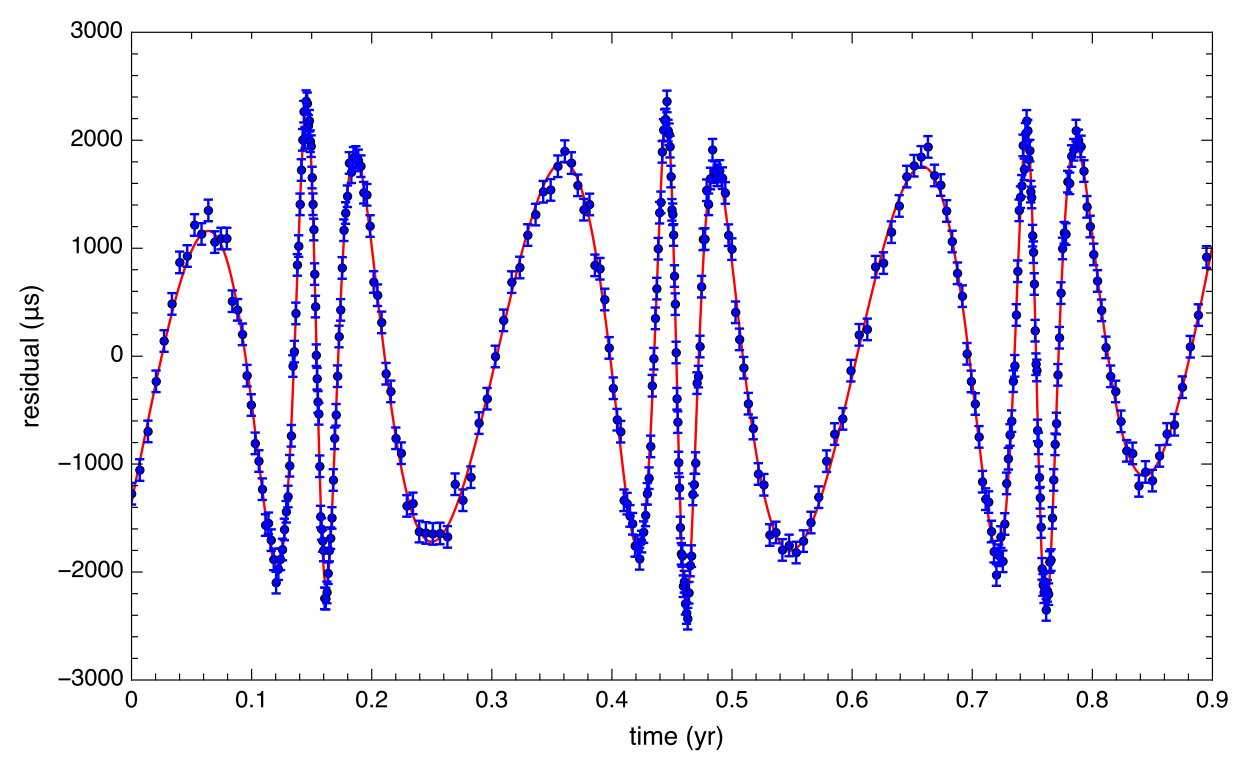

Figure 3: Characteristic pulsar timing residuals caused by the quadrupole moment of the $\operatorname{Sgr} \mathrm{A}^{*} \mathrm{BH}$ in simulated times-of-arrival (TOAs) over three orbital cycles (Liu et al. 2012). The orbital period is assumed to be $P_{\text {orb }}=0.3 \mathrm{yr}$ and the eccentricity $e=0.5$. The red line is the fitted pulsar timing model.

a pulsar orbiting Sgr A* in extremely short orbits $\left(P_{\text {orb }}<100 \mathrm{hr}\right)$. For instance, measurement of the relativistic apsidal advance, the second order Doppler shift, and the Shapiro delay (not shown) yield redundant constraints on the individual masses in the system as well as provide precision tests of GR. The Shapiro delay is detectable for elliptical orbits even when the orbit is face on. Geodetic precession of the spin axis will cause secular or periodic changes in measured pulse shapes if there is misalignment of the spin and orbital angular momenta, and secular orbital decay from gravitational radiation will be measurable for the more compact orbits $\left(P_{\text {orb }} \lesssim 10 \mathrm{hr}\right.$ ). Moreover, as Figure 4 demonstrates, only for these shortest orbital periods will the orbital decay time become so small that we will have little likelihood of detecting any such pulsar. Perhaps the most notable aspect of GC pulsars is that orbital perturbations are large enough so that even canonical $\left(10^{12} \mathrm{G}\right)$ pulsars with long periods ( $\gtrsim 0.5 \mathrm{~s}$ ), which ordinarily are considered to be noisy clocks, are suitable for making timing measurements. This means that any pulsar found in the GC is useful for the SKA pulsar program.

While many effects contribute to imprecision of TOAs (Cordes \& Shannon 2010), the dominant ones for GC pulsars with canonical periods will be intrinsic spin noise (Hobbs et al. 2010; Shannon \& Cordes 2010) and the large pulse widths that result from scattering broadening. With a conservative goal of about $1 \mathrm{~s}$ timing "precision" (in quotes because detection of gravitational waves with millisecond pulsars requires $<100 \mathrm{~ns}$ precision - Janssen et al. (2015)), many GR effects and orbital perturbations from stellar encounters can be measured one to two orders of magnitude better than the best IR interferometric measurements. The RMS spin noise for most objects will be below the $1 \mathrm{~s}$ target and the timing error from the pulsar width $W$ is $\delta t \sim W /(\mathrm{S} / \mathrm{N})$, where $\mathrm{S} / \mathrm{N}$ is the peak signal to noise ratio of the pulse. For widths $W<1 \mathrm{~s}$, it is reasonable to expect $\delta t \sim 0.01$ to $0.1 \mathrm{~s}$, providing even more precise orbital measurements.

At present, all these considerations rest on the assumption that the environment around Sgr 


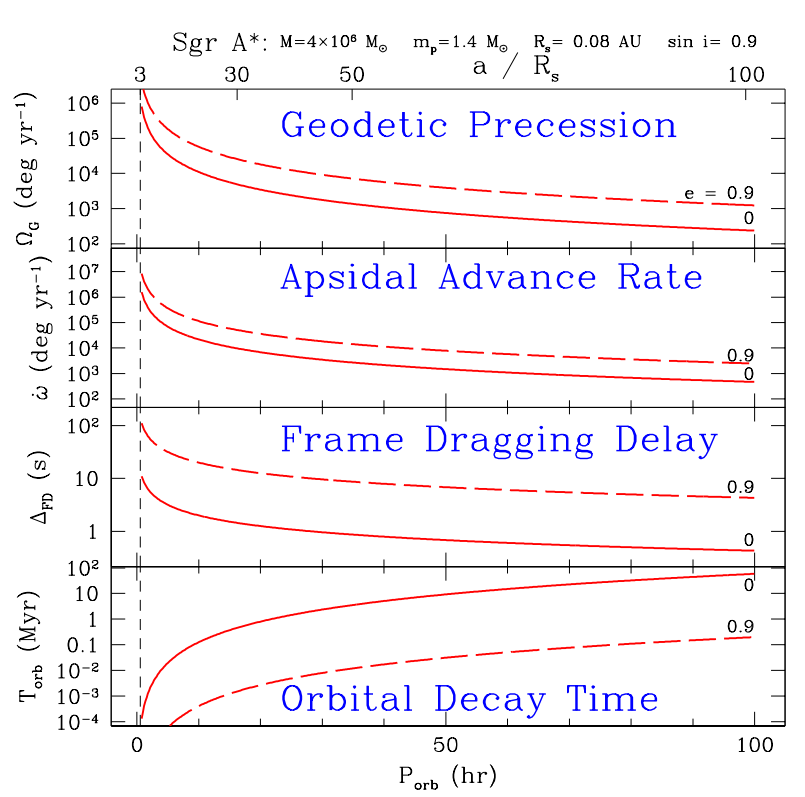

Figure 4: Relativistic effects that could be determined from measuring pulse TOAs from a pulsar closely orbiting Sgr A*. The bottom axis shows the orbital period, and the top axis shows the semi-major axis of the orbit, in units of the Schwarzschild radius of Sgr A*. All of the effects shown, except for frame dragging, have been detected in timing observations of binary pulsars. In all panels, the solid line indicates a circular orbit $(e=0)$ while the dashed line indicates a highly eccentric orbit $(e=0.9)$. For reference, the apsidal advance rates of PSR J0737-3039, B1913+16, and Mercury are too small to be plotted $\left(\dot{\omega}<20 \operatorname{deg} \mathrm{yr}^{-1}\right)$.

$A^{*}$ is sufficiently clean, i.e. external perturbations on the pulsar motion are negligible. As shown in Figure 1 the matter content of the GC consists not only of Sgr A* but early-type, late-type and evolved stars, dark matter, and magnetized plasma all of which will cause many perturbations that can be detected in pulsar TOAs. By measuring the Newtonian precession of elliptical orbits, estimation of the smoothly distributed mass, including both stars and dark matter, will be possible (Weinberg et al. 2005). In addition, strong constraints can be made on the density of stars from twobody perturbations of pulsar orbits (Weinberg et al. 2005; Merritt et al. 2010). To model potential external perturbations to TOAs, and see how they can be accounted for, or how much they degrade our capability to test GR, is subject to current research.

Chromatic effects from intervening magnetized plasmas are discussed in the next section; these effects are strongly frequency dependent and can be removed (after extracting all valuable scientific information on the properties of the GC interstellar medium) through appropriate multi-frequency measurements.

\section{Chromatic radio propagation effects}

Individual pulsar TOAs will show the well known dispersion and multipath delays caused by the integrated electron column density (the dispersion measure, DM) and by scattering from smallscale $(\lesssim 1$ au) density irregularities, characterized by the scattering measure (SM). Polarization measurements of pulse profiles will show Faraday rotation from which the rotation measure (RM) can be determined, thus constraining the integrated magnetic field.

Chromatic perturbations from magnetized plasma along the line of sight include the differential dispersion delay across a narrow bandwidth $\Delta v$ (in MHz), $\Delta t(v)=8.3 \mu \mathrm{sDM} \Delta v / v^{3}$, where $v$ is the observing frequency (in $\mathrm{GHz}$ ) and DM is the dispersion measure (in $\mathrm{pc}^{-3}$ ), which is the electron column density, $\mathrm{DM} \equiv \int d l n_{e}$. Such delays are removed from multi-frequency data both 
as part of the survey analysis (typically by the use of filterbanks) and also, with greater precision, during timing measurements.

Towards the GC, a more important factor is pulse broadening from scattering caused by fluctuations in electron density $\delta n_{e}$ that extend down to scales of a few hundred kilometers and have a roughly Kolmogorov wavenumber spectrum (Armstrong et al. 1995). For the strong scattering towards Sgr A*, first identified by angular diameter measurements of OH/IR masers and background AGNs, the pulse broadening has a time scale $\tau_{s} \propto D v^{\sim-4} \mathrm{SM}$, where $D$ is the pulsar distance and the scattering measure SM is the line of sight integral of $C_{n}^{2}$, the coefficient of the wavenumber spectrum for $\delta n_{e}$ (Cordes \& Lazio 2003). Unlike dispersion, which is easily removed to maximize the $\mathrm{S} / \mathrm{N}$ of a pulse, the deleterious effect of scatter broadening cannot be corrected for when many "scintles" 1 " are summed across the observing bandwidth, as they will be for GC pulsars.

Magnetized plasma Faraday rotates the linear polarization by an angle $v^{-2} \mathrm{RM}$ and is routinely measured for most pulsars using full Stokes parameter pulse shape measurements. In the GC this effect was demonstrated by measurements of the pulse polarization of the recently discovered magnetar, PSR J1745-2900, that revealed the second largest RM known in the Galaxy (the largest being that of Sgr A* itself) (Eatough et al. 2013a; Shannon \& Johnston 2013). From X-ray observations of the hot gas phase that Sgr $\mathrm{A}^{*}$ is accreting from (and which the magnetar is likely located in) a simple density profile was used to place limits on the strength of the magnetic field in this region; a value which can affect the dynamics of the accretion process onto the supermassive $\mathrm{BH}$ (Eatough et al. 2013a).

Once found, pulsars in the GC will therefore provide individual values of all three measures (DM, SM, and RM) for different lines of sight into the GC. Though not measured directly, the emission measure EM (the integral of $n_{e}^{2}$ ) can be related to DM and SM so as to constrain the "outer scale" of the wavenumber spectrum of $\delta n_{e}$ (Cordes \& Lazio 2002). The EM, while large toward Sgr $\mathrm{A}^{*}$, does not cause significant free-free absorption that would prohibit detection of pulsars as is clear since Sgr A* is seen through optically thin plasma down to at least $1 \mathrm{GHz}$.

\section{Finding and timing pulsars in the GC with the SKA}

\subsection{Past searches and updates on GC scattering}

Despite predictions that a large number of radio loud pulsars should be present in the GC (see Section 2.), at the time of writing just six are known within $0.5^{\circ}$ (Figure 2). It has been understood that the deficit in GC pulsars could be primarily explained by extreme scattering of radio waves in the GC (Cordes \& Lazio 1997; Lazio \& Cordes 1998; Cordes \& Lazio 2002, 2003). As mentioned in Section 4. scattering, which causes temporal broadening of pulses and a corresponding reduction in pulse $\mathrm{S} / \mathrm{N}$, has a strong frequency dependence $\left(\propto v^{\sim-4}\right)$. A clear mitigation strategy for dealing with pulse broadening due to scattering is to observe at frequencies much higher than typically used for pulsar searching. The significant constraint on this mitigation strategy is that typical pulsars have power-law spectra $\left(S_{v} \propto v^{\alpha}\right)$, usually with very steep spectra indices $(\langle\alpha\rangle \simeq-1.7)$ so their

\footnotetext{
${ }^{1}$ Scintles are the constructive interference patches seen in the frequency-time plane for nearby pulsars. The scattering towards Sgr A* is strong enough to prohibit measurement of individual scintles because they are narrower than typical frequency channel widths even at higher radio frequencies e.g. $14 \mathrm{GHz}$.
} 
flux densities decline rapidly with increasing frequency (Kramer et al. 1998). Fortunately, there is a subset of pulsars with relatively flat spectra. For example, of 293 pulsars with derived spectral indices in the ATNF catalog ${ }^{2}$ (Manchester et al. 2005), approximately 40 (or 14\%) have $\alpha>-1$. Moreover, a small number of pulsars have been detected at frequencies as high as $90 \mathrm{GHz}$ (Kramer et al. 1997; Morris et al. 1997). With such pulsars in mind, surveys of the GC at progressively higher radio frequencies have been performed (Klein et al. 2004; Johnston et al. 2006; Deneva et al. 2009; Deneva 2010; Macquart et al. 2010; Bates et al. 2011; Eatough et al. 2013b; Siemion et al. 2013) and resulted in the detection of five of the six pulsars displayed in Figure 2 (Johnston et al. 2006; Deneva et al. 2009); albeit at lower frequencies $\lesssim 3 \mathrm{GHz}$.

The sixth known GC pulsar, PSR J1745-2900, was first identified at X-ray wavelengths (Kennea et al. 2013; Mori et al. 2013) before detection in the radio (Eatough et al. 2013a; Shannon \& Johnston 2013). Recent measurements of the pulse broadening in this pulsar indicate the degree of scattering toward the GC is less than previously predicted (Spitler et al. 2014). In addition, measurements with the Very Long Baseline Array (VLBA) of the angular scatter broadening show that it has an angular size equivalent to Sgr A* itself. This suggests that PSR J1745-2900 and Sgr A* are behind the same scattering region. This fact coupled with the smaller pulse broadening time indicate that the scattering region (if modelled as a single thin screen of material) could be distant from the GC (Bower et al. 2014). However, the authors note that the paucity of GC pulsars still indicates that regions of intense scattering might exist in the GC. These regions could be of a more complex structure than in the simple picture described above (Spitler et al. 2014). Therefore high observing frequencies ( $\gtrsim 9 \mathrm{GHz}$ ) might still be necessary to detect GC pulsars.

In the following section we use the latest information about GC scattering to re-investigate the prospects for GC pulsar searches with the SKA.

\subsection{Prospects for GC pulsar searches with the SKA}

Our top goal is to find and then time radio pulsars within the central parsec (30" radius), with the highest priority placed upon pulsars as close as possible to Sgr A* . A secondary goal is to conduct a more complete census of the larger region $\left(\sim 30^{\prime}\right)$ in order to understand better the overall GC NS population. While the pulse broadening time due to scattering appears to be less than previously predicted, the effect is still large enough to necessitate pulsar searches of the GC at higher frequencies. In Figure 5 we show the detection sensitivity of pulsar periodicity searches of the GC performed with SKA1-MID and utilizing three of the proposed frequency bands outlined in (Dewdney et al. 2013). The figure shows the radio "pseudo luminosity" at $1.4 \mathrm{GHz}$ (given by $L_{\mathrm{p}}=S_{1400} D^{2}$, where $S_{1400}$ and $D$ are the period-averaged flux density at $1.4 \mathrm{GHz}$ and the distance respectively) versus the spin period. The dots are the actual values for known pulsars in the current (May 2014) ATNF catalog (Manchester et al. 2005). The curves plotted for each frequency band are derived from estimations of the minimum detectable flux density, $S_{\min }$, given by the radiometer equation as applied to pulsar observations,

$$
S_{\mathrm{min}}=\beta \frac{\mathrm{S} / \mathrm{N}_{\mathrm{min}} T_{\mathrm{sys}}}{G \sqrt{N_{\mathrm{pol}} \Delta v T_{\mathrm{obs}}}} \sqrt{\frac{W_{\mathrm{eff}}}{P-W_{\mathrm{eff}}}},
$$

\footnotetext{
${ }^{2}$ http://www.atnf.csiro.au/people/pulsar/psrcat/
} 
where $\beta$ is a digitization factor, $\mathrm{S} / \mathrm{N}_{\min }$ is the detection threshold, $T_{\text {sys }}$ is the system temperature, $G$ is the telescope gain (in units of $\mathrm{KJy}^{-1}$ ), $N_{\mathrm{pol}}$ is the number of polarization channels summed, $\Delta v$ is the bandwidth, $T_{\mathrm{obs}}$ is the observation time, $P$ is the pulsar spin period and $W_{\text {eff }}$ is the effective pulse width. Certain parameters have fairly standard values, namely, $\beta \approx 1, N_{\text {pol }}=2$, and $\mathrm{S} / \mathrm{N}_{\min } \approx 10$. The effective pulse width is given by the vector sum of the intrinsic pulse width and any unaccounted for pulse broadening effects in the following:

$$
W_{\mathrm{eff}}=\sqrt{\left(W_{50}^{2}+\tau_{\mathrm{samp}}^{2}+\tau_{\mathrm{DM}}^{2}+\tau_{\mathrm{s}}^{2}\right)}
$$

where $W_{50}$ is the intrinsic pulse width at half the pulse height (here assumed to be $0.05 P$ ), $\tau_{\text {samp }}$ is the data sampling interval, $\tau_{\mathrm{DM}}$ is the intra-channel dispersion broadening (in a filterbank) and $\tau_{\mathrm{s}}$ is the scatter broadening. The curves, which mark the minimum detectable pulsar spin period and luminosity, are plotted for pulsars at the distance of the GC and take into account the effects of scatter broadening, based on measurements of $\tau_{\mathrm{s}}$ from PSR J1745-2900 (Spitler et al. 2014), and assume a typical spectral index of $\alpha=-1.7$, thereby allowing the limits to be scaled to the equivalent sensitivity at $1.4 \mathrm{GHz}$. Further details of the numbers used in defining these curves are given in the caption to Figure 5.

If the scattering toward PSR J1745-2900 is representative of the inner GC as a whole Figure 5 shows that the common pulsars, with spin periods around $0.5 \mathrm{~s}$, might be detectable at frequencies as low as $2.4 \mathrm{GHz}$ (Band 3) with SKA1-MID. For mildly recycled pulsars, with spin periods from a few milliseconds to a few tens of milliseconds, $4 \mathrm{GHz}$ (Band 4) observations would be needed. Frequencies up to at least $9 \mathrm{GHz}$ (Band 5) might be required to detect the longer lived MSPs in the GC. As well as the possibility of detecting MSPs, GC pulsar surveys at higher frequencies offer the additional benefit of reduced background sky temperatures, improving sensitivity.

For comparison with current facilities a line for a $9 \mathrm{GHz}$ GC search with the Green Bank Telescope (GBT) has also been marked. The fact that a large fraction of the known pulsar population is already above this line might be of cause for concern for the prospects for finding more GC pulsars, however, (Chennamangalam \& Lorimer 2014) note that current non-detections from past GC surveys cannot yet rule out a large GC pulsar population which still need deeper surveys to be detected. SKA1-MID and the SKA are needed to address this problem.

Our search methodology consists of both periodicity searches, including correction for orbital motion, and searches for transient sources, because some pulsars are more easily detectable in single-pulse detection algorithms than in Fourier searches. In addition, the potential for discovering new classes of objects is maximized by searching for individual bursts.

Periodicity Searches: Periodicity searches are a standard tool for searching for pulsars and have been used for many years, in many directions, not limited to the GC. These algorithms include standard de-dispersion to remove dispersion delays, orbital demodulation, Fourier analysis of the resulting time series, and identification of signals above threshold, which requires classification as interference, instrumental contamination, or pulsars. For the GC, the most important aspect of algorithmic development concerns orbital demodulation, as it is for the shortest orbital period pulsars that the maximum GR effects can be measured. The simplest orbital demodulation is an acceleration search, which removes a parabolic signature 


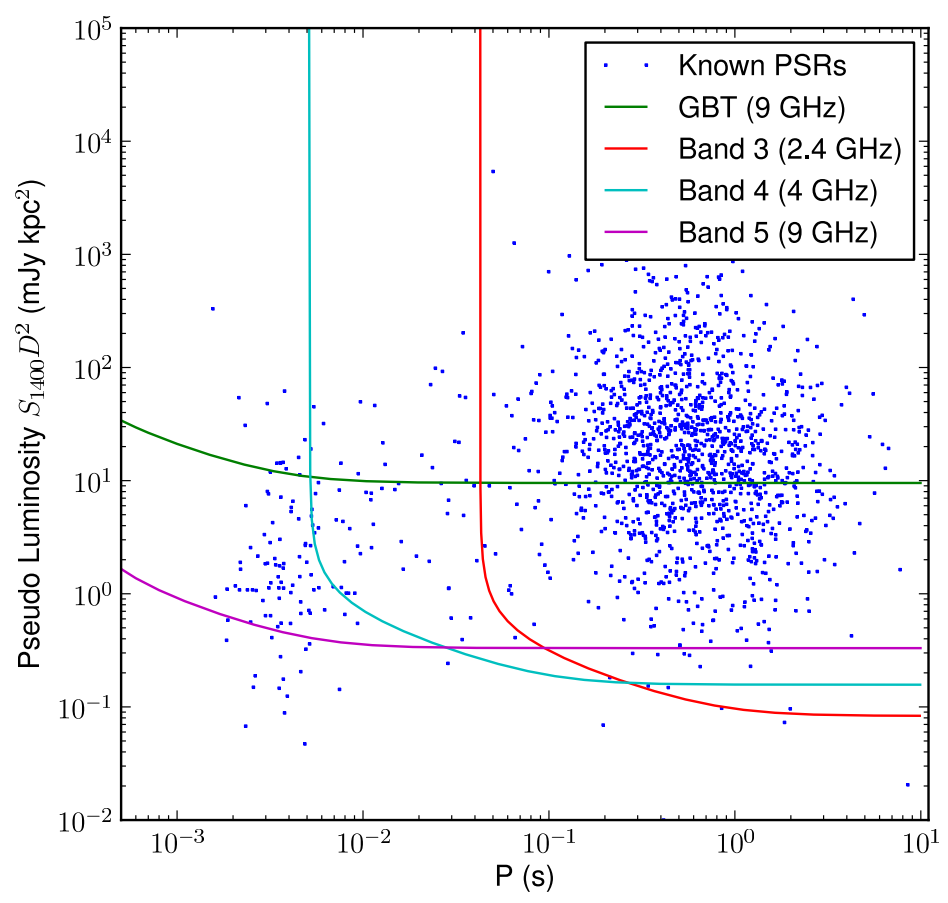

Figure 5: Detectability of pulsars in the GC via a periodicity search of a $6 \mathrm{hr}$ integration with the GBT at $9 \mathrm{GHz}$ and SKA1-MID at three of the proposed frequency bands. Points denote the period-averaged "pseudo luminosity" at $1.4 \mathrm{GHz}\left(L=S_{1400} D^{2}\right)$ versus spin period for known pulsars in the ATNF catalog (Manchester et al. 2005). The curves show sensitivity limits derived from the minimum detectable flux density (Equation 5.1). Pulsars are detectable if they are above a given curve. We have used a GC distance of $8.3 \mathrm{kpc}$; a $\mathrm{S} / \mathrm{N}=10$ detection threshold; a bandwidth $\Delta v=0.8 \mathrm{GHz}$ for the GBT, and bandwidths $\Delta v=1.4,2.4$ and 9.2 GHz for SKA1-MID Bands 3, 4 and 5 respectively (Dewdney et al. 2013); a system temperature, neglecting the effects background sky temperature, of $T_{\text {sys }}=27 \mathrm{~K}$; and a spectral index of $\alpha=-1.7$, while recognizing that some objects have flatter and some steeper spectra. Pulse broadening, $\tau_{s}$, values in the direction of Sgr A* are provided by measurements from the GG magnetar PSR J1745-2900 (Spitler et al. 2014). The curves will move lower for pulsars having spectra less steep than $v^{-1.7}$. The curves will also move lower if multiple integrations are combined (e.g., through incoherent combination of power spectra (Eatough et al. 2013b)). On the other hand, if for example the sensitivity of the core of SKA1-MID that is used for the pulsar search is reduced, by making the collecting area two thirds of the proposed size, the curves will move upward by an amount of $\log (1.5)$.

as an approximation to a Keplerian orbit. This approach works for data sets that have a duration which is a small fraction of the orbital period (Ransom et al. 2003).

Transient Searches: For compact orbits around Sgr A*, such as those $<100 \mathrm{hr}$ shown in Figure 4, the large GR effects that make such pulsars extremely attractive targets will also make them more difficult to detect. Fast geodetic precession, for example, may mimic an orbital period over a $6 \mathrm{hr}$ observation time and may be detectable at the output of an acceleration search. However, precession of the pulsar beam will make objects intermittent because the beam will precess out of our line of sight for a sizable fraction of the precession period. This 
effect has been seen in several of the NS-NS binaries, including the Hulse-Taylor pulsar (Kramer 1998). Consequently, single epoch search observations may be inadequate to find such sources. Moreover, some pulsars emit "giant" pulses, with strengths that are $1000 \times$ the mean pulse intensity (Hankins \& Rickett 1975; Hankins et al. 2003; Cognard et al. 1996; Johnston \& Romani 2003). In the case of the Crab pulsar, some giant pulses outshine the entire Crab Nebula (Hankins \& Rickett 1975), corresponding to brightness temperatures of order $10^{31} \mathrm{~K}$; (Hankins et al. 2003) observed "nano-giant" pulses having durations of only $2 \mathrm{~ns}$ and implied brightness temperatures of $10^{38} \mathrm{~K}$, by far the most luminous emission from any astronomical object.

Once GC pulsars are found with the SKA, our approach will be similar to that already used by e.g. (Deneva et al. 2009), namely they will be timed over short and moderate time periods to characterize their spin properties and any orbital motion. Each will be observed at multiple frequencies to find the optimal band for timing precision, which depends on period, pulse width, flux density and spectral index, and on the exact level of scattering. Depending on the object, we will then embark on a long-term timing campaign for measuring GR effects and probing the GC environment, with a cadence determined by the orbital period around Sgr A* and on any stellar orbital motion.

\section{References}

Armstrong, J. W., Rickett, B. J., \& Spangler, S. R. 1995, ApJ, 443, 209

Bates, S. D., Johnston, S., Lorimer, D. R., et al., 2011, MNRAS, 411, 1575B

Bower, G. C., Deller, A., Demorest, P., et al., 2014, ApJ, 780L, 2B

Bramante, J. \& Linden, T., 2014, Physical Review Letters, 113, 191301

Chennamangalam, J. \& Lorimer, D. R., 2014, MNRAS, 440, L86

Cognard, I., Shrauner, J. A., Taylor, J. H., \& Thorsett, S. E. 1996, ApJ, 457, L81

Cordes, J. M., \& Lazio, T. J. W. 1997, ApJ, 475, 557

Cordes, J. M., \& Lazio, T. J. W. 2002, arXiv:astro-ph/0207156

Cordes, J. M., \& Lazio, T. J. W. 2003, arXiv:astro-ph/0301598

Cordes, J. M., Kramer, M., Lazio, T. J. W., et al., 2004, New Astronomy Reviews, 48, 1413

Cordes, J. M. \& Shannon, R. M., 2010, ArXiv e-prints, arXiv:1010.3785

Deneva, J. S., 2010, PhD thesis, Cornell University

Deneva, J. S., Cordes, J. M., \& Lazio, T. J. W., 2009, ApJ, 702, L177

Dewdney, P. E., Turner, W., Millenaar, R., et al., 2013, "SKA1 System Baseline Design”, SKATEL-SKO-DD-001 Revision 1

Dexter, J. \& O’Leary, R. M. 2014, ApJ, 783, L7

Eatough, R. P., Falcke, H., Karuppusamy, R., et al. 2013a, Nature, 501, 391

Eatough, R. P., Kramer, M., Klein, B., et al. 2013b, IAU Symposium, 291, 382E

Eckart, A. \& Genzel, R. 1996, Nature, 383, 415

Eisenhauer, F., Perrin, G., Brandner, W., et al. 2011, The Messenger, 143, 16

Faucher-Giguère, C.-A, Loeb, A. 2011, MNRAS, 415, 3951

Ghez, A. M., Klein, B. L., Morris, M., \& Becklin, E. E. 1998, ApJ, 509, 678 
Ghez, A. M., Duchêne, G., Matthews, K., et al. 2003, ApJ, 586, L127

Ghez, A. M., Salim, S., Weinberg, N. N., et al. 2008, ApJ, 689, 1044

Gillessen, S., Eisenhauer, F., Fritz, T. K., et al. 2009a, ApJ, 707, L114

Gillessen, S., Eisenhauer, F., Trippe, S., et al. 2009b, ApJ, 692, 1075

Hankins, T. H., Kern, J. S., Weatherall, J. C., et al. 2003, Nature, 422, 141

Hankins, T. H. \& Rickett, B. J. 1975, Methods in Computational Physics. Volume 14 - Radio astronomy, 14, 55

Hobbs, G., Lyne, A. G., \& Kramer, M. 2010, MNRAS, 402, 1027

Janssen G. H., Hobbs G., McLaughlin M., et al. 2015, "Gravitational wave astronomy with the SKA," in proc. Advancing Astrophysics with the Square Kilometre Array, PoS(AASKA14)037

Johnston, S., Kramer, M., Lorimer, D. R., et al. 2006, MNRAS, 373, L6

Johnston, S. \& Romani, R. W. 2003, ApJ, 590, L95

Kennea, J. A., Burrows, D. N., Kouveliotou, C., et al. 2013, ApJ, 770, L24

Klein, B., Kramer, M., Müller, P., et al. 2004, IAU Symposium, 218, 133K

Kramer, M., Xilouris, K. M., Jessner, A., et al. 1997, A\&A, 322, 846

Kramer, M. 1998, ApJ, 509, 856

Kramer, M., Xilouris, K. M., Lorimer, D. R., et al. 1998, ApJ, 501, 270

Kramer, M., Backer, D. C., Cordes, J. M., et al. 2004, New Astronomy Reviews, 48, 993

Kramer, M., \& Stairs, I. H. 2008, ARA\&A, 46, 541

Lazio, T. J. W., \& Cordes, J. M. 2008, ApJS, 174, 481

Lazio, T. J. W., \& Cordes, J. M. 1998, ApJS, 118, 201

Liu, K., Wex, N., Kramer, M., et al. 2012, ApJ, 747, 1

Macquart, J.-P., Kanekar, N., Frail, D. A., \& Ransom, S. M. 2010, ApJ, 715, 939M

Manchester, R. N., Hobbs, G. B., Teoh, A. \& Hobbs, M. 2005, AJ, 129, 1993

Merritt, D., Alexander, T., Mikkola, S., \& Will, C. M. 2010, Physical Review D, 81, 062002

Meyer, L., Ghez, A. M., Schödel, R., et al. 2012, Science, 338, 84

Mori, K., Gotthelf, E. V., Zhang, S., et al. 2013, 770, L23

Morris, D., Kramer, M., Thum, C., et al. 1997, A\&A, 322, L17

Muno, M. P., Pfahl, E., Baganoff, F. K., et al. 2005, ApJ, 622, L113

Paczynski, B., \& Trimble, V. 1979, The Large-Scale Characteristics of the Galaxy, ed. W. B. Burton (Dordrecht: Reidel) p. 401

Pfahl, E., \& Loeb, A. 2004, ApJ, 615, 253

Ransom, S. M., Cordes, J. M., Eikenberry, S. S. 2003, ApJ, 589, 911

Reid, M. J., \& Brunthaler, A. 2004, ApJ, 616, 872

Sabha, N., Eckart, A., Merritt, D., et al. 2012, A\&A, 545, A70

Schödel, R., Ott, T., Genzel, R., et al. 2002, Nature, 419, 694

Seiradakis, J. H., Reich, W., Wielebinski, R., Lasenby, A. N., \& Yusef-Zadeh, F. 1989, A\&AS, 81, $291 \mathrm{~S}$

Shannon, R. M. \& Cordes, J. M. 2010, ArXiv e-prints, arXiv:1010.4794

Shannon, R. M. \& Johnston, S. 2013, MNRAS, 435, L29

Siemion, A., Bailes, M., Bower, G. 2013, IAU Symposium, 291, 57S

Spitler, L. G., Lee, K. J., Eatough, R. P., et al. 2014, ApJ, 780L, 3 S

Voss, R \& Gilfanov, M. 2007, MNRAS, 380, 1685 
Wang, Y., Creighton, T., Price, R. H., \& Jenet, F. A. 2009, ApJ, 705, 1252

Weinberg, N. N., Milosavljevic, M., \& Ghez, A. M. 2005, ApJ, 622, 878

Wex, N. \& Kopeikin, S. 1999, ApJ, 513, 388

Wex N., 2014, Brumberg Festschrift, arXiv:1402.5594

Wex, N., Gil, J., \& Sendyk, M. 1996, A\&A, 311, 746

Wharton, R. S., Chatterjee, S., Cordes, J. M., Deneva, J. S., Lazio, T. J. W. 2012, ApJ, 753, 108

Zhang, F., Lu, Y., \& Yu, Q. 2014, ApJ, 784, 106 